\title{
Los procesos de adquisición de conocimiento en la didáctica de la ciencia química en la educación superior
}

\author{
Margarita Rosa Rendón Fernández* \\ Pedro Nel Zapata Castañeda**
}

Artículo recibido: 18-08-2005 y aprobado: 26-04-2006

\begin{abstract}
Knowledge acquisition processes in chemistry teaching for undergraduate students
\end{abstract}

Resumen: El presente artículo muestra los resultados de una investigación realizada durante el año 2004 con un grupo de 25 estudiantes de primer semestre del programa de Ingeniería Ambiental de la Universidad de La Salle. La investigación se basó en un programa de intervención cognoscitiva en el que inicialmente se caracterizaron las habilidades cognoscitivas y metacognoscitivas relacionadas con los procesos de adquisición de conocimiento, todos ellos en el marco de la teoría triárquica de la inteligencia de Robert Sternberg (1985). Los resultados mostraron que cerca del $70 \%$ de los estudiantes, antes de la aplicación del programa de intervención, presentó dificultades en el empleo de los procesos de adquisición de conocimiento. La implementación del programa mejoró los procesos cognoscitivos y su conocimiento en más del $60 \%$ de los estudiantes de la población.

Palabras clave: Habilidades cognoscitivas y metacognoscitivas, paradigma de procesos, intervención cognoscitiva.
Abstract: This article shows the results of a research carried out with 25 undergraduate students attending environmental engineering classes of first semester at Universidad de La Salle during the year 2004. The investigation was based on a cognitive intervention program. First, the cognitive and Meta cognitive abilities that have to do with the processes of knowledge acquisition were characterized. All of them have been considered in the frame of thriarchic theory of intelligence from Robert Sternberg (1985).The results showed that almost $70 \%$ of the students presented difficulties in the use of the mentioned cognitive processes, before the application of the intervention program. The implementation of the program, improved the cognitive processes and the knowledge acquisition in more than $60 \%$ of the population's students.

Key words: Cognitive and meta cognitive abilities, processes paradigm, cognitive training.

* Investigadora principal. Profesora, Departamento de Ciencias Básicas Universidad de La Salle. Profesora catedrática, Universidad Pedagógica Nacional. mrendon@lasalle.edu.co.

** Coinvestigador. Profesor, Universidad Pedagógica Nacional. Profesor catedrático, Universidad de La Salle. pzapata@uni.pedagogica.edu.co 


\section{Introducción}

Sin duda alguna, para quienes hoy ejercen la profesión docente en los distintos niveles y modalidades del sistema educativo, las reflexiones acerca de la eficacia de su quehacer en el contexto actual de la enseñanza de las distintas ciencias posiblemente se relacionan, entre otros aspectos con la planeación y ejecución de actividades en el aula, la evaluación, las características de los contenidos disciplinares por desarrollar, las motivaciones e intereses de los estudiantes y los niveles de madurez y experiencia cognoscitiva de los estudiantes, necesarios para abordar las distintas tareas de aprendizaje.

Dentro de estos aspectos, recientemente han cobrado importancia los factores asociados al desarrollo de las habilidades de pensamiento de los estudiantes. No obstante, a pesar del creciente interés por estas habilidades, es poco lo que hoy sabemos acerca de su naturaleza y la forma como el sistema educativo puede promover su desarrollo.

La investigación aquí presentada, precisamente, se constituye en un esfuerzo sistemático por promover el desarrollo de las habilidades de pensamiento en el contexto educativo, fundamentada en el supuesto de que la aplicación de un modelo de enseñanza basado en procesos a una teoría que propone un concepto amplio e integral de inteligencia, debe originar una reestructuración profunda de los esquemas de pensamiento del individuo, siempre y cuando éste se someta a una práctica sistemática y rigurosa.

\section{Marco conceptual}

\subsection{Antecedentes}

Las principales investigaciones sobre intervención cognoscitiva a nivel mundial han sido referenciadas en la obra de Nickerson, Perkins y Smith (1994). Se destacan aquí los estudios sobre resolución de problemas, creatividad, metacognición, los programas o enfoques del "enseñar a pensar" y los programas de intervención en operaciones cognoscitivas como el Enriquecimiento instrumental de Feuerstein (1998) y el Proyecto de inteligencia Harvard (1994).

Dentro de los programas de intervención también se encuentran los enfoques de orientación heurística, como el patrón de solución de problemas, la enseñanza heurística de Schoenfeld (1980), los enfoques del pensamiento formal (Carmichael et al., 1980), y los enfoques de pensar sobre pensamiento por medio del lenguaje (Easterling y Pasamen, 1979), y la manipulación de símbolos, como el programa de filosofía para niños (Lipman et al., 1980).

Según Diez y Roman (1989), las formas tradicionales de intervención cognoscitiva suelen reducirse a dos: intervención en un test e intervención en un programa. En los primeros, se pretende ver las posibilidades de aprendizaje que posee un sujeto determinado. Para ello, a través de un diseño pretestintervención-postest, se comprueban los resultados positivos, y, a la vez, los principales errores cometidos en las respuestas más significativas del pretest. Se seleccionan aquellos errores más representativos de los estudiantes y se entrena a los sujetos para no volverlos 
a cometer. Posteriormente se aplica un postest y se miden las diferencias entre el pretest y el postest.

La intervención en un programa pretende desarrollar el potencial de aprendizaje (Feuerstein, 1998) o el "enseñar a pensar" (Nickerson, Perkins y Smith, 1994). Los programas más representativos son los de intervención cognoscitiva. La duración de la aplicación de los programas de intervención cognoscitiva suele depender del curso escolar, con intervenciones varias veces a la semana. Los objetivos por alcanzar casi siempre están bien definidos y son indicadores de la posible modificabilidad cognoscitiva que se prentende conseguir.

En el campo de la teoría triárquica de la inteligencia de R. Sternberg y el paradigma de procesos, el trabajo llevado a cabo por la doctora Margarita Amnestoy (1998) en el Instituto Tecnológico y de Estudios Superiores de Monterrey se constituyó en la principal fuente de referencia para la presente investigación. El trabajo dirigido por ella consta de cinco cursos destinados a desarrollar las habilidades de pensamiento mediante la estimulación de procesos cognoscitivos.

\subsection{La teoría triárquica de la inteligencia de R. Sternberg}

Según la doctora Amnestoy (1998), "la teoría triárquica de la inteligencia humana es una propuesta mediante la cual se presenta un concepto ampliado de la inteligencia. Consta de tres subteorías: componencial, experiencial y contextual" (p. 8).

La subteoría componencial relaciona la inteligencia con el mundo interior del individuo, e identifica los mecanismos que regulan la conducta inteligente. Está integrada por tres tipos de procesos mentales: los metacomponentes, que determinan cómo se planifican, supervisan y evalúan las acciones; los componentes de ejecución, que definen los procesos mentales de uso más frecuente para facilitar la ejecución de acciones previamente planificadas, y los componentes de adquisición de conocimiento, integrados por procesos dirigidos a optimizar el aprendizaje con base en la información que proporciona el contexto.

La subteoría componencial especifica los mecanismos mentales de la conducta inteligente, y parte del principio de que aun cuando los individuos difieran en la ejecución al resolver problemas, dichos mecanismos desde el punto de vista conceptual son, en general, los mismos, en todos y para todos los individuos, independientemente de sus condiciones sociales y culturales.

Por otra parte, refiriéndose a la teoría experiencial, la doctora. Amnestoy ha señalado que ésta:

...especifica el momento de la vida y experiencia del individuo en el cual la inteligencia está más plena y activamente relacionada con la realización de tareas y la solución de problemas. Se refiere especialmente a la acción inteligente y plantea los tipos de problemas referidos al tratamiento de situaciones novedosas y la automatización de los procesos mentales (p. 9).

En la subteoría contextual, según esta autora:

La inteligencia se relaciona con el mundo exterior del individuo, y se identifican las tres actividades que caracterizan la conducta inteligente, a saber: la adaptación al ambiente, la 
selección de éste y su transformación. Se destaca la importancia de la relación del sujeto con el medio ambiente en la determinación de lo que constituye una conducta inteligente en un ámbito o contexto dado (p. 9).

\subsection{Componentes de adquisición de conocimiento}

La capacidad de aprender y de utilizar el lenguaje es la característica que diferencia a la especie humana de cualquier otro ser viviente. El vocabulario de un adulto incluye decenas de miles de palabras, y las diferencias entre una y otra persona estriban en factores relacionados con la cultura, la educación y las características individuales. También se sabe que sólo una pequeña parte del vocabulario de una persona es producto de la enseñanza directa; la mayoría de sus adquisiciones procede del aprendizaje de significados a partir del contexto donde se le presentan las palabras.

Según Amnestoy (1998):

El estudio de los procesos utilizados para descifrar significados de palabras a partir del contexto ha revelado conocimientos útiles para desarrollar técnicas de enseñanza y aprendizaje cuya finalidad es mejorar las habilidades verbales de los estudiantes para descifrar y comprender significados mediante la información del contexto de palabras conocidas. Las dificultades en el aprendizaje de conceptos verbales dependen de dos tipos de factores, a saber: a) del grado de facilitación que provee el medio de la situación de aprendizaje, y b) de las habilidades que posea el aprendiz para utilizar las variables que afectan la adquisición de conocimientos a partir de la descontextualización de las palabras (p. 381).

\subsection{El paradigma de procesos}

El paradigma de procesos explica los aspectos conceptuales y metodológicos de un enfoque sobre el pensamiento basado en la teoría del procesamiento de información, en la generación de esquemas o estructuras cognoscitivas y en el desarrollo del metaconocimiento, como fuentes de autoaprendizaje. El conocimiento se define como la información acerca de hechos, conceptos, principios, reglas y planteamientos conceptuales o teóricos que conforman una disciplina o un campo de estudio o, simplemente, en el ámbito de lo cotidiano, la información incidental acerca de hechos o eventos del mundo que rodea al individuo. Los procesos son operaciones de pensamiento capaces de transformar una imagen o representación mental en otra o en una actividad motora.

Según la doctora Amnestoy (1998):

La enseñanza basada en procesos consiste en aplicar el enfoque de procesos en la metodología para estimular el aprendizaje. Los pilares del modelo de procesos para desarrollar habilidades intelectuales son: la intencionalidad del acto mental y de la actividad mediante la cual se dirige y optimiza el uso de la capacidad intelectual del individuo; la concienciación del acto mental implícito en el proceso, el enfoque de sistemas como instrumento de pensamiento, como producto de la metodología de procesos y como fuente de retroalimentación y de optimización, la participación activa del estudiante como medio que permite verificar procesos y productos del pensamiento y seguir el progreso de la persona, el monitoreo de los procesos y la función del profesor como mediador del procedimiento enseñanza aprendizaje (p. 8). 
En general, la propuesta de la doctora Amnestoy para el desarrollo de habilidades de pensamiento puede ser incluida bien sea dentro de los enfoques de intervención en operaciones cognoscitivas o bien dentro de los enfoques de orientación heurística.

\section{Delimitación y formulación del problema}

Con relación al problema de la investigación adelantada, diversos estudios han mostrado un detrimento de las habilidades intelectuales en estudiantes que cursan la educación básica primaria, secundaria y superior, siendo mucho más notable en estos dos últimos niveles (Hederich, 1989; Gardner, 1991). Por tanto, es posible, a manera de hipótesis, que la mayor parte de los estudiantes colombianos presente las mismas carencias en cuanto a las habilidades intelectuales puesto que, por lo regular, en nuestro medio educativo no se ha hecho un especial énfasis en el desarrollo de tales habilidades.

Con base en lo anterior, se plantearon dos problemáticas sobre las cuales la ejecución de la investigación pretendió construir conocimiento. La primera se refiere al conjunto de características que debe reunir un programa de intervención cognoscitiva para que incida de forma eficiente y eficaz en el desarrollo de habilidades de pensamiento relacionadas con los procesos de adquisición de conocimiento. La segunda problemática está vinculada específicamente con la naturaleza misma de las habilidades estudiadas y su presencia en la población objeto de estudio.

\section{Metodología}

En términos generales, la investigación se fundamentó en un diseño de series cronológicas del tipo $\mathrm{O}_{1} \mathrm{X} \mathrm{O}_{2} \mathrm{X} \mathrm{O}_{3} \mathrm{X} \mathrm{O}_{4}$, sin grupo control, en el que $\mathrm{O}_{1}, \mathrm{O}_{2}, \mathrm{O}_{3} \mathrm{y}$ $\mathrm{O}_{4}$ representan diversas observaciones a través del tiempo, y X la estrategia o programa de intervención desarrollado. La población objeto de estudio estuvo conformada por 25 estudiantes, de los cuales el 76\% (19) eran mujeres y el 24\% (6), hombres. Las edades de los estudiantes de la población oscilaron entre los 17 y los 22 años.

En general, la ejecución del proyecto se puede describir a través de las siguientes etapas.

1. Aplicación de una prueba pretest general sobre los procesos de adquisición de conocimiento.

2. Análisis de resultados de la prueba pretest.

3. Aplicación del programa de intervención cognoscitiva: diseño y ejecución de actividades orientadas a favorecer el desarrollo de los procesos de pensamiento relacionados con la adquisición de conocimiento.

4. Evaluación de los procesos de pensamiento a medida que se avanza en el programa de intervención cognoscitiva.

\subsection{Diseño de técnicas de recolección de información}

Para la evaluación de los procesos de adquisición de conocimiento, se presentaron a los estudiantes párrafos cortos en cada uno de los cuales se sustituyó una palabra por un vocablo desconocido (véase anexo 1). Funda- 
mentalmente se examinó si los estudiantes podían identificar el significado de la palabra desconocida en cada uno de ellos.

\subsection{Características generales del programa de intervención en los procesos de adquisición de conocimiento}

El desarrollo del programa de intervención se realizó a lo largo de tres temáticas disciplinares de la ciencia química, a saber: las propiedades periódicas de los elementos químicos, los estados de agregación de la materia y las soluciones químicas. Una vez aplicadas las pruebas pretest, se analizaron los resultados, y con base en éstos se determinaron aquellos procesos de pensamiento en donde los estudiantes tuvieron mayores dificultades.

Para el desarrollo del programa, al iniciar se entregó a cada uno de los estudiantes un manual completo que contenía una explicación de todos los procesos de pensamiento estudiados (Guía del alumno). En esta guía, cada proceso estudiado parte de una justificación, unos objetivos por lograr y las actividades que debe realizar. La figura 1 ilustra el programa de intervención cognoscitiva.

En general, en el programa de intervención cognoscitiva sobre los procesos de adquisición de conocimiento realizado con los estudiantes se hizo énfasis en los siguientes aspectos:

- Para descifrar el significado de una palabra desconocida en un texto se debe:
a) separar la información relevante de la irrelevante en el contexto,
b) combinar la información seleccio- nada en un todo significativo, y
c) utilizar la experiencia previa para relacionar el contexto de la palabra desconocida con algo familiar.

- Estos procesos se identifican respectivamente con los siguientes nombres: codificación selectiva, combinación selectiva y comparación selectiva, y se denominan procesos de adquisición de conocimiento.

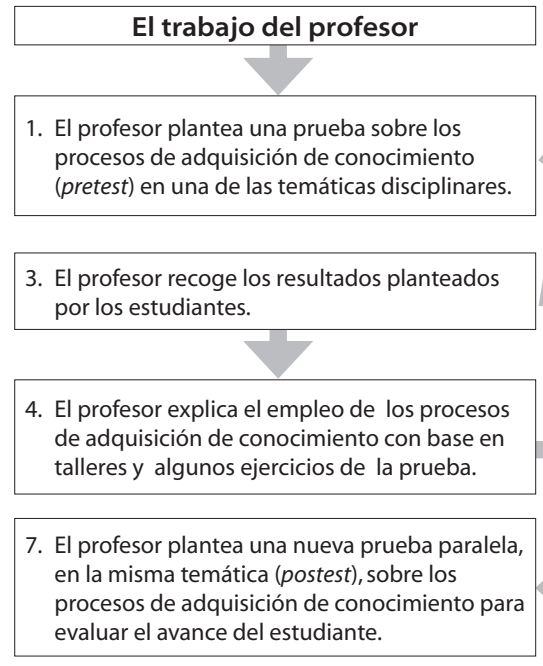

Figura 1. Programa de intervención cognoscitiva.
El trabajo del estudiante
2. El estudiante resuelve la prueba individualmente.
5. El estudiante identifica los errores y aciertos cometidos en la solución de las pruebas y en el empleo de los procesos de adquisición de conocimiento.
6. El estudiante corrige los errores.


- La codificación selectiva es el proceso mediante el cual se separa la información relevante de la irrelevante. Se sabe que cuando se trata de descifrar el significado de una palabra desconocida a partir del contexto, las señales relevantes que permiten establecer la asociación de la palabra con hechos o situaciones conocidas están mezcladas con información irrelevante que interfiere y obstaculiza la tarea. La separación de estos dos tipos de información es el primer paso en este sistema de aprendizaje de palabras.

- La codificación selectiva implica dos procesos, a saber: la combinación selectiva, mediante la cual se integran las señales relevantes ya codificadas en una definición razonable de la palabra desconocida, y la comparación selectiva, que permite relacionar la información adquirida a partir del contexto con la que se tiene almacenada como resultado de conocimientos y experiencias previos. El propósito de esta operación es darle sentido a la nueva información, y para lograrlo se busca cómo relacionarla con conocimientos previamente almacenados en la memoria.

- Las variables mediadoras son variables que afectan la aplicación de los procesos de adquisición de conocimiento a las señales contextuales. A continuación se caracterizan siete variables mediadoras, que fueron aplicadas por los estudiantes en el análisis de los distintas pruebas y talleres realizados durante el programa de intervención:
- Numero de veces que aparece la palabra desconocida en el fragmento.

- Variedad de los contextos asociados a la palabra desconocida.

- Importancia de la palabra desconocida para la comprensión del material en el cual aparece.

- Utilidad del contexto circundante para comprender el significado de una palabra desconocida.

- El número de palabras desconocidas incluidas en el fragmento.

- El carácter concreto de la palabra desconocida y de su contexto.

- Utilidad de la experiencia.

\section{Resultados y análisis de resultados} Inicialmente se muestran los resultados obtenidos en la prueba pretest general y los respectivos análisis realizados. Posteriormente, los resultados obtenidos en las distintas pruebas pretest y postest en cada una de las temáticas de la ciencia química abordadas durante el desarrollo del programa de intervención cognoscitiva.

\subsection{Prueba pretest general sobre los procesos de adquisición de conocimiento}

En general, los resultados de esta prueba -la cual sirvió como pretest del tema de propiedades periódicas- mostraron que la mayor parte de los estudiantes no logró identificar el significado de las palabras desconocidas en los distintos fragmentos de texto presentados. 


\begin{tabular}{ccccccc} 
& & \multicolumn{3}{c}{ Ítem } & \multirow{2}{*}{ Total grupo } \\
\cline { 2 - 5 } & $\mathbf{1}$ & $\mathbf{2}$ & $\mathbf{3}$ & $\mathbf{4}$ & 13 \\
\hline $\mathrm{n}$ Total & 3 & 8 & 2 & 0 & 13 \\
\hline $\mathrm{p}(\%)$ & 11 & 31 & 11 & 0,0 & 87 \\
\hline
\end{tabular}

Tabla 1. Resultados prueba pretest general, procesos de adquisición de conocimiento en puntuación bruta y porcentaje según estudiantes de la población. Propiedades periódicas $(\mathrm{N}=25)$.

Los valores $p$ y $q$ en las tablas indican, respectivamente, el porcentaje de estudiantes que aprueban y desaprueban cada uno de los ítems de las pruebas.

Los resultados obtenidos en la prueba pretest general se clasificaron en las siguientes categorías:

a) Estudiantes que no contestaron los ítems de la prueba. En estos casos la no contestación, lo mismo que la contestación errónea, muestran la dificultad de los estudiantes para hallar el significado de las palabras desconocidas (puntuación 0).

b) Estudiantes que contestaron pero no lograron identificar el significado de las palabras desconocidas (puntuación 0).

c) Estudiantes que lograron identificar el significado de las palabras desconocidas (puntuación 1).

Como se observa en la tabla 1 , en general, más del 70\% de los estudiantes no pudo identificar el significado de los cuatro vocablos desconocidos en los textos presentados. Ahora bien, aunque algunos estudiantes lograron identificar, en algunos casos, el significado de palabras desconocidas, ninguno mostró el empleo sistemático de las señales contextuales, la codificación, la combinación y la comparación selectiva.
Parece evidente que los estudiantes no hacen un esfuerzo por utilizar la información que proporciona el contexto en el que se encuentra la palabra desconocida para hallar su significado y, más aún, no utilizan los conocimientos previos que ya poseen para relacionar la información que proporciona el párrafo con lo que ya posiblemente saben.

En síntesis, estos resultados mostraron la necesidad de adelantar el programa de intervención cognoscitiva en los procesos de adquisición de conocimiento con todos los estudiantes de la población.

\subsection{Resultados de las pruebas sobre procesos de adquisición de conocimiento según los estudiantes de la población durante el programa de intervención cognoscitiva}

Una vez aplicada la prueba pretest general sobre los procesos de adquisición de conocimiento, y analizados sus resultados se desarrolló el programa de intervención cognoscitiva con base en la guía del alumno y los talleres en los que se buscó la aplicación de estos procesos a la lectura de textos de química con vocablos desconocidos. Los resultados obtenidos en las distintas pruebas se describen a continuación. 


\subsubsection{Los procesos de adquisición de conocimiento en el tema propiedades periódicas}

En la tabla 2 se describen los resulta- la población en el postest en el tema de dos obtenidos por los estudiantes de propiedades periódicas.

\begin{tabular}{|c|c|c|c|c|}
\hline \multirow{2}{*}{ Estudiante } & \multicolumn{3}{|c|}{ Ítem } & \multirow{2}{*}{$\begin{array}{l}\text { Total } \\
\text { grupo }\end{array}$} \\
\hline & 1 & 2 & 3 & \\
\hline Total & 22 & 16 & 15 & 53 \\
\hline$p(\%)$ & 88 & 64 & 60 & 71 \\
\hline$q(\%)$ & 12 & 36 & 40 & 29 \\
\hline
\end{tabular}

Tabla 2. Resultados prueba postest sobre procesos de ejecución de conocimiento según estudiantes de la población. Propiedades periódicas ( $\mathrm{N}=25)$.

En la tabla 3 se comparan los desempeños de los estudiantes de la población en las pruebas pretest y postest sobre los procesos de adquisición de conocimiento en esta temática.

\begin{tabular}{ccc}
\hline Desempeño & No. & $\%$ \\
\hline Mejoraron & 25 & 100 \\
\hline Desmejoraron & 0 & 0 \\
\hline Igual & 0 & 0 \\
\hline
\end{tabular}

Tabla 3. Comparación del desempeño en los procesos de adquisición de conocimiento en pruebas pretest y postest según número y porcentaje de estudiantes de la población. Prop iedades periódicas ( $\mathrm{N}=25$ ).

Como se observa en la tabla 3 , los resultados muestran que el $100 \%$ de los estudiantes mejoró en los procesos de adquisición de conocimiento. Algunos estudiantes en el postest lograron identificar el significado de todos los vocablos desconocidos mientras que otros lograron identificar uno o dos de ellos.

En este caso, en los postest se observa que los estudiantes identifican algunas señales contextuales en cada uno de los textos presentados. Estos resultados reflejan el efecto de la experiencia previa en la comprensión selectiva, pues los estudiantes, al desarrollar el postest, ya han tenido previamente la influencia no sólo del taller sobre el proceso de adquisición de conocimiento en esta temática, sino también de la didáctica y los contenidos propios del curso de química en el que se encuentran. Este conocimiento previo permite a los estudiantes "descifrar" más fácilmente el significado de los vocablos desconocidos.

Por otra parte, debe señalarse que muchos de los conceptos que se trabajan en la ciencia química son "abstractos" para los estudiantes pues no están al alcance de experiencias concretas en las que el significado de los conceptos es más fácil de inferir. Conceptos 
como "electronegatividad", "electrones", "energía de ionización", aunque son muy específicos, son bastante abstractos, lo que limita en ocasiones la utilidad de la experiencia concreta para la abstracción de su significado.

Finalmente, debe señalarse que los textos presentados a los estudiantes son relativamente breves, por lo que esta característica puede afectar la capacidad de algunos estudiantes para descifrar el significado de los vocablos, ya que la información suministrada en el texto puede no ser suficiente.

\subsubsection{Los procesos de adquisición de conocimiento en el tema estados de agregación}

A continuación se describen los resultados obtenidos por los estudiantes en los procesos de adquisición de conocimiento en el tema de estados de agregación de la materia.

\begin{tabular}{ccccc}
\hline & & Ítem & \multicolumn{2}{c}{$\begin{array}{c}\text { Total } \\
\text { grupo }\end{array}$} \\
\cline { 2 - 5 } & $\mathbf{1}$ & $\mathbf{2}$ & $\mathbf{3}$ & 39 \\
\hline $\mathrm{p}(\%)$ & 19 & 7 & 13 & 52 \\
\hline $\mathrm{q}(\%)$ & 76 & 28 & 52 & 48 \\
\hline
\end{tabular}

Tabla 4. Resultados prueba pretest sobre procesos de adquisición de conocimiento según estudiantes de la población. Estados de agregación de la materia ( $\mathrm{N}=25$ ).

\begin{tabular}{|c|c|c|c|c|}
\hline \multirow{2}{*}{ Estudiante } & \multicolumn{3}{|c|}{ Ítem } & \multirow{2}{*}{ Total grupo } \\
\hline & 1 & 2 & 3 & \\
\hline Total & 16 & 21 & 15 & 52 \\
\hline $\mathrm{p}(\%)$ & 64 & 84 & 60 & 69 \\
\hline$q(\%)$ & 36 & 16 & 40 & 31 \\
\hline
\end{tabular}

Tabla 5. Resultados prueba postest sobre procesos de adquisición de conocimiento según estudiantes de la población. Estados de agregación de la materia ( $\mathrm{N}=25)$.

En la tabla 6 se comparan los desempeños en las pruebas pretest y postest en esta temática.

\begin{tabular}{ccc}
\hline Desempeño & No. & $\%$ \\
\hline Mejoraron & 13 & 52 \\
\hline Desmejoraron & 5 & 20 \\
\hline Igual & 7 & 28
\end{tabular}

Tabla 6. Comparación del desempeño en los procesos de adquisición de conocimiento en pruebas pretest y postest según número y porcentaje de estudiantes de la población. Estados de agregación de la materia. 
Los resultados de la tabla 6 indican que el desempeño en el pretest en el tema de estados de agregación fue mejor que el obtenido en el pretest inicial en el tema de propiedades periódicas, lo que indicaría que a medida que se avanza en el programa de intervención, los estudiantes tienden a mejorar sus resultados en las diversas pruebas.

Al examinar los resultados mostrados en la tabla 6 se observa que el $52 \%$ de los estudiantes mejoró en la prueba postest en el tema de estados de agregación, mientras que un $20 \%$ desmejoró. De los estudiantes que obtuvieron puntuaciones iguales en el pretest y en el postest, se observa que cuatro obtuvieron puntuaciones de $100 \%$ en ambas pruebas y dos obtuvieron puntuaciones de $66 \%$ en ambas pruebas, lo que indicaría que estos estudiantes utilizan los procesos de adquisición de conocimiento de forma más sistemática, estable y con mejores resultados que otros estudiantes de la población.

\subsubsection{Los procesos de adquisición en el tema soluciones químicas}

A continuación se describen los resultados obtenidos por los estudiantes en los procesos de adquisición de conocimiento en el tema soluciones químicas.

\begin{tabular}{ccccc}
\hline \multirow{2}{*}{ Estudiante } & \multicolumn{1}{c}{ Ítem } & \multirow{2}{*}{ Total grupo } \\
\cline { 2 - 4 } & $\mathbf{1}$ & $\mathbf{2}$ & $\mathbf{3}$ & 19 \\
\hline Total & 12 & 1 & 6 & 29 \\
\hline $\mathbf{p ~ ( \% ) ~}$ & 54 & 4 & 27 & 71 \\
\hline
\end{tabular}

Tabla 7. Resultados prueba pretest sobre procesos de adquisición de conocimiento según estudiantes de la población. Soluciones químicas.

\begin{tabular}{ccccc}
\hline \multirow{2}{*}{ Estudiante } & \multicolumn{1}{c}{ Ítem } & \multicolumn{2}{c}{ Total grupo } \\
\cline { 2 - 5 } & $\mathbf{1}$ & $\mathbf{2}$ & $\mathbf{3}$ & 54 \\
\hline Total & 22 & 23 & 9 & 75 \\
\hline $\mathbf{p ~ ( \% ) ~}$ & 92 & 96 & 37 & 25 \\
\hline
\end{tabular}

Tabla 8. Resultados prueba postest sobre procesos de adquisición de conocimiento. Soluciones químicas.

Los desempeños en las pruebas pretest y postest en esta temática se comparan en la tabla 9.

\begin{tabular}{ccc}
\hline Desempeño & No. & $\%$ \\
\hline Mejoraron & 17 & 68 \\
\hline Desmejoraron & 2 & 8 \\
\hline Igual & 3 & 12 \\
\hline No comparados & 3 & 12 \\
\hline
\end{tabular}

Tabla 9. Comparación del desempeño en los procesos de adquisición de conocimiento en pruebas pretest y postest según número y porcentaje de estudiantes de la población. Soluciones químicas. 
Como se observa en la tabla 9, tres estudiantes de la población no pudieron ser comparados por no presentar una de las pruebas. Por otra parte, el 68\% de los estudiantes mejoró en la prueba postest y los tres estudiantes que permanecieron igual obtuvieron puntuaciones en el prestest y en el postest del 66\%, lo que indica, en general, que cerca del $60 \%$ de los estudiantes pudieron emplear los procesos de adquisición de conocimiento para descifrar el significado de los vocablos desconocidos en los textos presentados.

Por otra parte, estos resultados también reflejarían algunas dificultades de los estudiantes para elaborar inferencias a partir de la lectura. Así, pues, es un proceso natural deducir más allá del material realmente presentado (Clark, 1977; Graesser y Bower, 1990). Las inferencias realizadas durante la comprensión cumplen dos funciones generales: por un lado, permiten establecer relaciones dentro del nuevo material y entre esta nueva información y el conocimiento ya existente en la memoria. Esto facilita la integración del nuevo material en las representaciones de la memoria de la información previamente aprendida y también contribuye a proporcionar cierta organización y estructura a la información. En segundo lugar, las inferencias "rellenan las ranuras" vacías (lagunas) en la estructura global.

Una cuestión teórica difícil en el estudio de las inferencias es cómo determinar cuál de todas las posibles inferencias se generarán en línea mientras se lee o se escucha. La hipótesis minimalista (McKoon y Ratcliff, 1992) sugiere que las únicas inferencias que se codifican automáticamente son aquellas necesarias para establecer coherencia local en el texto. Por otro lado, la teoría construccionista (Graesser y cols., 1994) sostiene que generamos en línea tanto las inferencias que conectan con el texto como las extratextuales.

Craik y Lockhart (1972) afirman que los recuerdos difieren en cuanto al grado de procesamiento que reciben. Su teoría sobre los niveles de procesamiento asume que la información puede ser procesada en diferentes niveles. Una información puede ser procesada en un nivel superficial, poco profundo, o puede ser interpretada en un nivel más profundo. Estos autores sugieren que cuanto más procesamiento recibe la información, más duradera será su huella en la memoria y mayor la probabilidad de que sea recordada.

Según Craik y Tulving (1975), la elaboración de la información se refiere a su grado de relación con otros acontecimientos. Crack y Tulving sugieren que si bien se analizan tanto los rasgos físicos como los semánticos de la información, hay diferencias en cuanto a los niveles de elaboración fonética y semántica.

\subsection{Análisis general del programa de intervención cognoscitiva}

A efectos de proporcionar un marco de referencia que posibilite una mejor comprensión del programa sobre desarrollo de habilidades de pensamiento, se presenta a continuación un análisis sobre los principales aspectos que -a juicio de los autores- pudieron intervenir o no en los resultados obtenidos.

\subsubsection{Efecto de variables ambientales}

Como se señaló anteriormente, la investigación se llevó a cabo en el propio 
ambiente educativo en donde realizan su actividad los estudiantes. Para el desarrollo del programa no se preparó ningún ambiente físico especial más que el proporcionado por el propio salón asignado a los estudiantes. Igualmente, no se emplearon recursos adicionales aparte de la guía del alumno y los talleres entregados a cada uno de los estudiantes de la población. En este caso, se considera que las características de los espacios físicos u otras variables de tipo ambiental no afectaron los resultados obtenidos por los estudiantes en cada una de las pruebas, ni tampoco el desarrollo del programa de intervención por parte del profesor.

\subsubsection{Efecto de variables conexas a las variables investigadas}

El programa de intervención se realizó con estudiantes que se encuentran entre los 16 y 20 años de edad, que poseen el nivel de madurez cognoscitiva y de experiencia propios de esa edad. Es posible, a manera de hipótesis, que existan diferencias en la incidencia de un programa de intervención sobre los procesos de pensamiento en función de factores como la edad; sin embargo, la investigación no permite concluir nada al respecto debido a la similitud de las edades de los estudiantes de la población.

La doctora Amnestoy ha recomendado llevar a cabo el programa de intervención con estudiantes mayores de 14 años; no obstante, parece más importante el nivel de desarrollo cognoscitivo que la propia edad, a menos que estén fuertemente relacionados.

\subsubsection{Efecto de variables derivadas de la actuación del investigador}

El desarrollo del programa de intervención, así como la valoración dada a las distintas pruebas pretest y postest en cada una de las etapas de la investigación, fue llevado a cabo por el propio director de la investigación. No se puede desconocer por tanto la intencionalidad del acto educativo y el hecho de procurar en todos los aspectos la eficacia del programa de intervención, algo acerca de lo cual los estudiantes que participaron en el proyecto conocían desde el inicio. Es decir, los estudiantes conocían desde el principio la naturaleza de la investigación y las intenciones del investigador.

La puntuación de las pruebas pretest y postest se realizó siguiendo los mismos criterios; sin embargo, esto no asegura eliminar diversos sesgos debidos a la percepción, y sus límites, del investigador. En todo caso, las pruebas y las distintas actividades realizadas durante todo el programa de intervención se llevaron a cabo desde el marco conceptual que sustenta el proyecto con el fin de procurar su coherencia.

\subsubsection{Efecto de variables derivadas de la presencia del investigador en el campo de la investigación}

Durante el desarrollo de la investigación se considera que no hubo el establecimiento de relaciones sociales diferenciales de simpatía o antipatía que pudiesen incidir en los resultados. No obstante, se observó, en algunos casos, estudiantes que manifestaron en algún momento su cansancio frente a algunas de las actividades y la aplicación reiterada de diversas pruebas. Es posible que estas 
situaciones hubiesen generado antipatía hacia el investigador y el programa de intervención por parte de algunos estudiantes que pudiera haber afectado los resultados de las pruebas y la colaboración hacia el desarrollo del programa. En todo caso, se buscó incentivar más la participación de los estudiantes que tuvieron algunas dificultades procurando evitar su aislamiento del curso.

\subsubsection{Efecto de variables derivadas de la forma de actuación y respuesta de los sujetos investigados}

En esta investigación se considera que no influyeron en los resultados variables como el respeto social, la aprehensión evaluativa, las características de la demanda y la dependencia de la memoria.

En cuanto al respeto social, es evidente que la investigación no trata sobre aspectos o situaciones frente a los cuales los estudiantes tuviesen que disfrazar sus respuestas. Por el contrario, se invitó siempre a los estudiantes a diligenciar las pruebas de la forma más honesta posible, procurando en todo momento utilizar sus procesos de pensamiento para resolverlas.

En lo relativo a la aprehensión evaluativa, se observó la particularidad de ligar los resultados del programa de intervención a la evaluación académica de los estudiantes. En este caso fue necesario explicar a los estudiantes que los resultados de las pruebas se emplearían como criterio para su evaluación. No obstante, la evaluación académica de los estudiantes comprendió aspectos referentes no sólo al resultado de las pruebas, sino al desempeño de los estudiantes en las distintas sesiones, dentro de una concepción más integral de evaluación.

En cuanto a las características de la demanda, los estudiantes conocían de antemano las intenciones de la investigación y de cada una de las pruebas, pero se considera que este conocimiento no alteró los resultados obtenidos por ellos, pues parece poco probable pensar que de este conocimiento se puede lograr súbitamente un mejor rendimiento en las pruebas, las cuales son de eficiencia más que de opinión o de personalidad.

Finalmente, con relación a la dependencia de la memoria, las pruebas y cada uno de los ítems, dadas sus características, no dependen de la memoria en sentido estricto sino de otras habilidades relacionadas con procesos de pensamiento. Sin embargo, no se puede desconocer que aun dichos procesos puedan hacer parte de la memoria procedimental, semántica o incluso episódica. De todas formas, en las distintas pruebas se incluyeron ítems en donde los estudiantes deberían obtener la información a partir de un análisis del contexto más que acudir a la memoria. Se podría afirmar, por ejemplo, que incluso los procesos directivos necesitan ser "memorizados" para emplearlos luego en la solución de problemas, lo cual resulta evidente. No obstante, la incorporación de dicha información en la memoria no es un proceso arbitrario y sin sentido, sino que implica procesos complejos mediante los cuales la información adquiere significado en virtud de su utilidad para resolver problemas, construir inferencias o adquirir nuevo conocimiento. 


\subsubsection{Efectos de las variables ligadas al tiempo} Puesto que en la investigación se empleó un diseño de series cronológicas, sin grupo control, es posible analizar el efecto de algunos factores como la historia, la maduración y el efecto reactivo de los test y la situación experimental en los resultados obtenidos.

En cuanto a la historia, debe señalarse que la diferencia de tiempo entre la aplicación de los pretest y los postest, en cada una de las etapas, fue menor de un mes, tiempo en el cual, aparte de la influencia del ambiente escolar y los ambientes intrafamiliares y extrafamiliares propios de los estudiantes, se considera que no hubo influencia de factores que incidieran específicamente en los procesos de pensamiento y en los resultados obtenidos, más que el programa de intervención.

Con relación al efecto de la maduración, bien sea biológica o cognitiva, se asume que el tiempo tan breve entre las pruebas pre y postest no permiten el influjo de estas variables.

Respecto a los efectos reactivos de los test, tanto los pretest como los postest eran muy similares en su estructura y en el tipo de demanda en cada uno de los ítems. Se podría pensar que los pretest pudieron entrenar a los sujetos en la contestación de los postest; sin embargo, el diseño empleado no posibilita evaluar este efecto ya que las estrategias de intervención se fundamentaron en el desarrollo de cada uno de los ítems de los test en los que se encontraron dificultades, por lo que los mejores resultados de algunos estudiantes en el postest no pueden explicarse en función del entrenamiento en la contestación a partir del pretest.
Por otra parte, es posible que emplear dos pruebas de la misma estructura posibilite que los estudiantes se familiaricen con la prueba, y esto pueda influir, por ejemplo, en la velocidad con que se diligencian los postest, más que en la naturaleza de las contestaciones a cada uno de los ítems.

En cuanto a los efectos de la estrategia, los resultados señalados en las distintas tablas mostrarían que para el caso de aquellos estudiantes que avanzaron en cada una de las habilidades de pensamiento estudiadas, tal situación se debió al influjo del programa de intervención.

\section{Conclusiones}

- En general, los resultados de las pruebas diagnósticas empleadas mostraron que antes de desarrollar el programa de intervención cognoscitiva, más del $70 \%$ de los estudiantes de la población no evidenció dominio de los distintos procesos de adquisición de conocimiento, lo que confirma la primera hipótesis de la investigación.

- Los resultados de las distintas pruebas postest aplicadas indicaron que el programa de intervención mejoró no sólo la utilización de los procesos de adquisición de conocimiento, sino el conocimiento acerca de ellos en más del 60\% de los estudiantes de la población. Estos resultados confirman la segunda hipótesis planteada sobre la influencia del programa de intervención en el desarrollo de los procesos de pensamiento.

- La organización del programa de intervención en lecciones con justificación, objetivos, actividades y 
reflexiones posibilita un mayor control sobre la estrategia, aunque en algunas ocasiones puede constituirse en un protocolo que restringe y lleva a los estudiantes al cansancio y a una especie de falta de atención estratégica que dificulta el desarrollo de los procesos de pensamiento. Adicionalmente, puede decirse que aunque el tiempo dedicado al estudio de cada uno de los procesos en cada sesión puede ser suficiente, es necesario un esfuerzo deliberado y sistemático por parte de los estudiantes para ejercitar dichos procesos aun fuera del contexto escolar.

- Durante el programa de intervención, los estudiantes emplean, con distintos grados de eficacia, los procesos de adquisición de conocimiento para el razonamiento y la comprensión de textos. En este sentido, la investigación confirma la subteoría sobre la naturaleza de estos procesos y la forma como éstos pueden relacionarse para razonar y actuar efectivamente sobre el mundo que rodea al sujeto que aprende. $\boldsymbol{\Delta}$

\section{Anexo 1}

\section{Pretest inicial procesos de adquisición de conocimiento}

En los textos que aparecen a continuación, identificar el término químico que sustituye cada una de las palabras desconocidas en el texto.

1. Generalmente la PREA disminuye al aumentar el tamaño atómico. Mientras más grande sea el átomo, el electrón más débilmente unido estará más lejos del núcleo; por tanto, se necesita menos energía para arrancarlo. Así, la PREA es inversa al tamaño atómico: aumenta en los periodos y disminuye en los grupos.

Término químico que sustituye la palabra PREA

2. Los FREDOS se caracterizan por su tamaño pequeño, alta energía de iotización y alta electronegatividad. Como necesitan muy pocos electrones para completar su último subnivel, tienen tendencia a recibir electrones dando iones negativos. Con los metales forman enlaces iónicos; también reacciones entre sí para formar compuestos covalentes que contienen dos o más átomos. Al hacer estas combinaciones sus electrones quedan todos generalmente apareados y localizados en las moléculas individuales, razón por la cual no conducen la electricidad.

Término químico que sustituye la palabra FREDOS

3. Los GUMIS tienen ciertas propiedades totalmente diferentes de las otras sustancias. Son sólidos cristalinos de estructura simple, cuyas unidades son átomos de la misma clase. Estudios de rayos $X$ demuestran que los GUMIS forman cristales que se pueden considerar como conjuntos de esferas empaquetadas de tal manera que muchas de ellas se ponen en contacto tanto como sea posible. Existen tres estructuras geométricas de cristalización de los GUMIS. Dos de éstas se conocen como compactas porque permiten que los átomos se junten lo más cerca posible unos de otros. La otra es la denominada cúbica centrada en el cuerpo que es de estructura abierta.

Término químico que sustituye la palabra GUMIS 
4. Un DOMO no produce nuevas sustancias. Sólo altera la forma o el estado de agregación (fase) de la sustancia. EI DOMO de una sustancia sirve para determinar algunas de sus propiedades. El DOMO se puede determinar sin que ocurra reacción de la sustancia. El DOMO está directamente relacionado con la estructura de la materia.Todo DOMO va acompañado siempre de un cambio de energía.

Término químico que sustituye la palabra DOMO

\section{Bibliografía}

Amnestoy, M. (1998). Desarrollo de habilidades de pensamiento. Procesos directivos, ejecutivos y de adquisición de conocimiento. México: Trillas.

Carmichael, J. W. et al. (1980). "Project SOAR". En: The American biology teacher, 42 (3), 169-173.

Clark, H. H. (1977). "Inferences in comprehension. En D. Laberge y S. J Samuels (eds.), Basic Processes in reading: Perception and comprehension. Hillsdale, N. J.: Erlbaum de Vega (1984).

Craik, F .I. M. y Lockhart, R. S. (1972). "Levels of processing: A framework for memory research". Journal of verbal learning and behaviour, 11: 671-684.

$y$ Tulving, E. (1975). "Depth of processing and the retentions of words in episodic memory". Journal of experimental psychology: General, 104: 268-294.

Diez, L. E. y Roman, P. M. (1989). "Intervención cognitiva y mejora de la inteligencia”. En: Revista de Educación No. 289, p 391-408.

Easterling, J. y Pasamen, J. (1979). Confront, construct, complete: a comprehensive approach to writing. New Jersey: Hayden Book.

Feuerstein, R. y Jackson, Y. (1998). "Feuerstein's IE and structural cognitive modifiability”. En: R. Samuda (ed.), Advances in cross cultural assessment. Thousand Oaks, CA: Sage.
Gardner, H. (1991). The unschooled mind. Nueva York: New York based book.

Graesser, A. C. Bower, G. H. (1990). Inferences and text comprehension. San Diego: Academia Press.

Singer, M. y Trabaos, T. (1994). "Constructing inferences during text comprehension. Psychological review, 101: 371-395.

Harvard (1994). Miguel Mejía Fernández, coord. "Proyecto de Inteligencia Harvard". $2^{\mathrm{a}}$. ed. CEPE, S.L.

Hederich, C. M. (1989). "Inteligencia en Colombia. Un elemento en la búsqueda de identidad”. En: Revista Colombiana de Educación. №. 20. Bogotá: Universidad Pedagógica Nacional.

Lipman, M. et. al. (1980). Philosophy in the classroom. Philadelphia: Temple.

McKoon, G. y Ratcliff, R. (1992). "Inference during reading". Psychological review, 99: 440-466.

Nickerson, R., Perkins, D. y Smith, E. (1994). Enseñar a pensar. Aspectos de la aptitud intelectual, 3a. ed. (Trad. Barcelona, Paidós/ MEC, 1987).

Schoenfeld, A. H. (1980). "Teaching problem solving skills". American mathematical montly, 87(10), 794-805.

Sternberg, R. (1985). Triarchic theory of human intelligence. Nueva York: Cambridge University Press. 